\title{
Noninvasive Retinal Angiographic Parameters for Cardiovascular Risk Assessment: A New Insight into "Old Classics"
}

\author{
Ryo Kawasaki ${ }^{1,2}$
}

${ }^{1}$ Artificial Intelligence Center for Medical Research and Application, Osaka University Hospital, Osaka, Japan

${ }^{2}$ Department of Vision Informatics, Osaka University Graduate School of Medicine, Osaka, Japan

\section{See article vol. 28: 000-000}

In the paper published by Zhong et al. ${ }^{1)}$, new modalities of optical coherence tomography angiography (OCTA) can provide a risk evaluation for coronary artery disease $(\mathrm{CAD})$ in patients with suspected angina. OCT is a new imaging modality that can visualize the retinal vasculature in detail in capillary networks and multilayered en face images without using a fluorescein dye. Zhong et al. reported that they developed a multivariable logistic regression model to predict the presence of CAD in 795 participants, including 508 participants with suspected angina due to CAD. They used a sparse modeling method of the least absolute shrinkage and selection operator (LASSO) to select important imaging characteristics from a pool of imaging characteristics captured in OCTA images and successfully built a model with the relatively small number of participants. Most importantly, the authors compared the predictive power of OCTA with that of ECG, a most common diagnostic modality used in daily practice. As a result, OCTA parameters demonstrated good discrimination ability against ECG and additional predictive power independent of ECG; a combination of ECG and OCT performed best compared with the mode including each.

The findings of the study by Zhong et al. have a great potential to overcome the shortcomings of the traditional retinal vascular assessment in the context of cardiovascular disease risk prediction. Morphological changes in the retinal vasculature, e.g., a retinal arteriolar narrowing or arterio-venous crossing signs, have been considered candidates of such biomarkers since Keith, Wegener, and Barker reported the predictive value of the retinal vascular changes, clearly stratifying the risk of mortality into four groups.
Indeed, the retina provides a unique window to inspect blood vessels noninvasively and evaluate vascular health in vivo.

From a historical viewpoint, Japan has been a unique country practicing this concept into a health screening program nation-wide, i.e., developing nonmydriatic fundus cameras and utilizing them in a nationwide cardiovascular screening program since the 1970 s to 2000s. The wide use of fundus photography and retinal vascular assessment has decreased because of the primary target of the health check-up programs changing to the metabolic syndrome. At the same time, there were criticisms regarding the clinical aspect that retinal vascular changes are only capturing severe hypertensive organ damage and it may not hold its predictive value as a biomarker as most of the persons are categorized into mild stage due to the lifestyle changes and improved medical care for hypertension in the last few decades. Also, regarding the methodological aspect, an inter- and intra-rater agreement has been questioned, as the subjective inspection to determine the retinal signs requires vast experience and passion for graders, and most physicians and ophthalmologists have the least training for this purpose. As a result, there is a need for quantitative assessment of retinal vascular changes that can capture earlier changes, with high reproducibility, and ideally, automated gradings would be preferable so that the retinal vascular assessment can contribute to this era. Recent studies have linked semiquantitative measurement of vessel caliber size to cardiovascular diseases ${ }^{2,3)}$. However, it is limited in research use and not fully incorporated into any screening program as it takes time and workload.

In this context, using OCTA parameters is a fascinating idea as it is noninvasive and the parameters are quantitative and can be obtained automatically. There has been a tremendous amount of research

Address for correspondence: Ryo Kawasaki, Artificial Intelligence Center for Medical Research and Application, Osaka University Hospital, Osaka, Japan E-mail:.kawasaki@ophthal.med.osaka-u.ac.jp

Received: May 7, $2021 \quad$ Accepted for publication: May 23, 2021

Copyright@2021 Japan Atherosclerosis Society

This article is distributed under the terms of the latest version of CC BY-NC-SA defined by the Creative Commons Attribution License. 
work aiming to identify a cardiovascular biomarker that reflects preclinical vascular health. Such a biomarker would help physicians practice effective risk stratification to identify persons at high risk of cardiovascular events, preventive measures to reduce the risk, and evaluate the effects of interventions as a surrogate marker of devastating events. An ideal biomarker should be measured repeatedly, noninvasively, less costly, and most importantly, be reflecting a preclinical pathological state. The next step of this work would definitely be an external validation of these findings in larger datasets. It would also be necessary to compare the performance, costeffectiveness, and accessibility against nontraditional risk factor candidates such as coronary artery calcium (CAC), the ankle-brachial index (ABI), and highsensitivity C-reactive protein (hsCRP). It is also interesting if the retinal vascular parameters can be a surrogate marker of intervention to mitigate the cardiovascular risk load. Another direction of utilizing retinal images is a prediction tool based on a deep learning model without extracting morphological features ${ }^{4)}$. However, using OCTA parameters might still have superiority in its interpretability and explainability compared with a deep learning model.

Overall, this study provides further steps to bring the classic concept of direct microvascular assessment, and with emerging technological advancements, it will open up vast potentials of using the retinal vasculature to improve the prediction and management of cardiovascular diseases.

\section{Conflict of Interest}

The author received clinical research funding from Senju Pharmaceutical Co. Ltd., and endowed department by Topcon Co. Both are not related to the current contents.

\section{References}

1) Zhong P, Qin J, Li Z, Jiang L, Peng Q, Huang M, Lin Y, Liu B, Li C, Wu Q, Kuang Y, Cui S, Yu H, Liu Z, Yang X. Development and Validation of Retinal Vasculature Nomogram in Suspected Angina Due to Coronary Artery Disease. J Atheroscler Thromb, 2021; in press. doi: https://doi.org/10.5551/jat.62059

2) Wong TY, Kamineni A, Klein R, Sharrett AR, Klein BE, Siscovick DS, Cushman M, Duncan BB. Quantitative retinal venular caliber and risk of cardiovascular disease in older persons: the cardiovascular health study. Arch Intern Med, 2006; 166: 2388-2394

3) Kawasaki R, Xie J, Cheung N, Lamoureux E, Klein R, Klein BE, Cotch MF, Sharrett AR, Shea S, Wong TY; MESA. Retinal microvascular signs and risk of stroke: the Multi-Ethnic Study of Atherosclerosis (MESA). Stroke, 2012; 43: 3245-3251

4) Poplin R, Varadarajan AV, Blumer K, Liu Y, McConnell MV, Corrado GS, Peng L, Webster DR. Prediction of cardiovascular risk factors from retinal fundus photographs via deep learning. Nat Biomed Eng, 2018; 2: 158-164 